\title{
O Uso de Internet e Redes Sociais e a Relação com Indícios de Ansiedade e Depressão em Estudantes de Medicina
}

\author{
The Use of the Internet and Social Networks and \\ the Relationship with Symptoms of Anxiety and \\ Depression among Medical Students
}

\author{
Maíra Sandes Moromizato \\ Danilo Bastos Bispo Ferreira ${ }^{I}$ \\ Lucas Santana Marques de Souza \\ Renata Franco Leite ${ }^{I}$ \\ Fernanda Nunes Macedo ${ }^{I}$ \\ Déborah Pimentel ${ }^{I}$
}

\section{PALAVRAS-CHAVE}

- Estudantes de Medicina.

- Internet.

- Rede Social.

- Dependência.

- Ansiedade.

- Depressão.
RESUMO

Introdução: A internet é uma ferramenta imprescindível na atualidade, pois possibilita acesso fácil e rápido às informações e a manutenção de laços afetivos por meio das redes sociais. Entretanto, quando se percebe um uso descontrolado e desadaptativo, ocorre a chamada adicção por internet (AI). Estudos prévios investigaram diversas comorbidades associadas a esse transtorno, gerando um importante conhecimento importante para a conduta clínica. Objetivo: Investigar a correlação entre indicadores do uso de internet e redes sociais e a presença de sintomas ansiosos e depressivos. Métodos: Estudo descritivo, transversal, quantitativo, com amostragem por conveniência, realizado com estudantes de Medicina no ano de 2015. Os sintomas ansiosos e depressivos foram analisados por meio do Inventário de Ansiedade de Beck (BAI) e do Inventário de Depressão de Beck (BDI), respectivamente. Além disso, utilizou-se um questionário confeccionado pelos pesquisadores com base no Internet Addiction Test (IAT). Resultados: Dos 169 estudantes que participaram da pesquisa, 98,8\% (167) fazem uso diário de internet e/ou redes sociais. Foi avaliada a prevalência de diversos indícios do uso prejudicial da internet, bem como a concepção dos participantes sobre seu uso. Não foi encontrada associação estatística entre o tempo gasto na internet e a presença de sintomas ansiosos e depressivos com os escores BAI e BDI. Contudo, foram percebidas algumas associações estatisticamente significativas com os resultados dos escores BAI e BDI tanto com indicadores da necessidade de verificação da internet quanto com indicativos do uso desadaptativo da internet. Conclusão: O presente estudo ratifica achados prévios na literatura que apontam que a AI não está necessariamente relacionada com o tempo gasto na internet, mas com o padrão desadaptativo do uso. Os resultados aqui encontrados podem servir de base para futuras intervenções em instituições de ensino que busquem minimizar o prejuízo desse transtorno cada vez mais presente. 


\section{KEYWORDS \\ - Medical Students. \\ - Internet \\ - Social Networks \\ - Dependency. \\ - Anxiety. \\ - Depression.}

Recebido em: 09/01/2017

Aprovado em: 26/09/2017

\section{ABSTRACT}

Introduction: The internet is now an essential tool, through which people enjoy easy and rapid access to information and can maintain personal relationships through social networks. However, when its use becomes uncontrolled and maladaptive, what can entail is known as Internet Addiction (IA). In previous studies, several comorbidities associated with this disorder have been investigated, generating knowledge that is important for clinical management. Objective: To investigate the correlation between indicators of internet use and social networks with the presence of symptoms of anxiety and depression. Methods: A descriptive, cross-sectional, quantitative study with convenience sampling, carried out with medical students in 2015. The anxiety and depression symptoms were analyzed through the Beck Anxiety Inventory (BAI) and the Beck Depression Inventory (BDI), respectively. In addition, a questionnaire made by the researchers based on the internet Addiction Test (IAT) was used. Results: Out of the 169 students who participated in the research, 98.8\% (167) use the internet and/or social networks on a daily basis. The prevalence of various indications of the harmful use of the internet was evaluated, as well as the participants' conception of its use. No statistical association was found between the time spent on the internet and the presence of symptoms of anxiety and depression, according to the BAI and BDI scores. However, some statistically significant associations were observed between the results obtained from the BAI and BDI and indicators of the need for internet verification as well as signs of maladaptive use of the internet. Conclusion: The present study ratifies the previous findings in the literature by pointing out that IA is not necessarily related to the time spent on the internet, but rather to a maladaptive pattern of use. The results found here may serve as basis for future interventions in educational institutions that seek to minimize the damage of this disorder, which has become increasingly present.

\section{INTRODUÇÃO}

A internet se tornou uma ferramenta extremamente necessária na vida atual. $\mathrm{O}$ acesso rico à informação, à comunicação instantânea e ao entretenimento fez crescer exponencialmente o número de usuários da web nos últimos anos, que chegou a 2,5 bilhões em todo o mundo, tendo como grupo majoritário adolescentes e adultos jovens ${ }^{1,2}$. Paralelamente aos benefícios, emergem os efeitos prejudiciais do uso de forma desadaptativa e a Adicção por Internet (AI), considerada uma epidemia do século XXI, digna de preocupação como um problema mundial de saúde mental ${ }^{2-5}$.

Em muitos casos se percebe somente o uso excessivo da internet, que consiste no gasto de tempo exagerado, de forma descontrolada e consumindo o tempo necessário para outras atividades do indivíduo. A AI é caracterizada pelo uso com padrão desadaptativo, levando a danos clinicamente significativos ou sofrimento emocional ${ }^{1}$.

Adicção por internet, dependência, uso patológico, vício ou uso problemático são termos utilizados como sinônimos na literatura para nomear esse uso desadaptativo ${ }^{2,6}$. Esse transtorno é descrito como uma preocupação intensa com o uso da internet, uso compulsivo, gasto de tempo excessivo na web, inabilidade para manejar esse tempo, considerando ainda o mundo sem internet desinteressante, irritabilidade no caso de ser interrompido quando está conectado e diminuição dos relacionamentos sociais por causa desse $\mathrm{uso}^{2,7}$.

São diversos os efeitos deletérios relacionados à $\mathrm{AI}$, como alterações na qualidade do sono, na nutrição e na atividade física, menor desempenho acadêmico ou profissional e prejuízo nos relacionamentos interpessoais. Além disso, diversas pesquisas relacionam a $\mathrm{AI}$ aos transtornos de humor, transtorno de déficit de atenção e hiperatividade, transtorno de uso de substâncias, ansiedade, ansiedade social, solidão, baixa autoestima, menores níveis de atividade física, hostilidade e comportamento agressivo, comportamento compulsivo, impulsividade, maiores taxas de transtornos de personalidade, menor felicidade e vitalidade subjetivas, prejuízos na saúde mental de forma geral e suicídio $2,3,5-8$.

A ansiedade e a depressão são encontradas comumente em estudantes de Medicina9,10. Entretanto, no Brasil e na América Latina, de forma geral, não foram realizados muitos estudos sobre o uso da internet e a relação com problemas de saúde mental. Dessa forma, é escasso o conhecimento sobre o tema em nosso meio, o que acabou por subestimar seu real im- 
pacto nos pacientes. Além disso, investigar as correlações entre as comorbidades psiquiátricas e a AI pode ajudar a entender e a tratar melhor esta última em pacientes com o quadro ${ }^{5}$.

Para suprir a lacuna do conhecimento, o presente estudo associa a presença de sintomas ansiosos e depressivos com indícios do uso problemático da internet em estudantes de Medicina de uma universidade particular de uma capital brasileira. Os resultados aqui encontrados podem ajudar a esclarecer essas relações e alertar sobre a prevenção do uso problemático da internet entre estudantes universitários de forma geral.

\section{METODOLOGIA}

Estudo descritivo, transversal, quantitativo, com amostragem por conveniência, realizado com estudantes de Medicina de uma universidade privada na cidade de Aracaju, no Estado de Sergipe.

Essa universidade teve a primeira turma de Medicina no ano de 2010. Portanto, no momento da coleta de dados, os alunos cursavam o décimo primeiro período. $\mathrm{O}$ curso adota a metodologia ativa de ensino (Problem Based Learning - PBL) e foi pontuado com nota quatro, num escore máximo de cinco, na única avaliação realizada pelo MEC.

Ao todo, havia cerca de 450 estudantes matriculados no curso de Medicina, dos quais 169 participaram da pesquisa. A abordagem dos estudantes ocorreu durante os intervalos das atividades curriculares, nas dependências da universidade ou nos locais de estágio. Os critérios de exclusão foram: idade menor que 18 anos e não estar matriculado no curso da referida universidade.

Foram utilizados como instrumentos para a coleta de dados três questionários autoaplicáveis. O primeiro foi desenvolvido pela equipe desta pesquisa com base no Internet Addiction Test (IAT) do pesquisador Kimberly Young ${ }^{11}$, composto por questões objetivas. Também foram usados o Inventário de Ansiedade de Beck (BAI) e o Inventário de Depressão de Beck (BDI), cada um com 21 questões com 4 itens e com pontuações que variam de 0 a 3 , formando escores máximos de 63 pontos.

Para realizar a análise estatística, as variáveis foram testadas quanto à normalidade dos dados por meio do teste de Shapiro-Wilk e foram apresentadas por meio de mediana (Md) e seus quartis (25\%-75\%) por não assumirem o pressuposto de normalidade. As variáveis categóricas foram apresentadas em frequências absoluta e relativa. Para a análise de comparação entre as idades de homens e mulheres, foi aplicado o teste de Mann-Whitney. As análises de associação foram realizadas por meio do teste do Qui-Quadrado ou Exato de Fisher. A significância estatística foi estipulada em $5 \%(p \leq 0,05)$. Para todas as análises foi usado o programa Statistical Package for the Social Sciences (SPSS 15.0). As associações estatísticas que utilizaram o BAI e o BDI consideraram como ponto de corte a pontuação $\geq 11$ para $\mathrm{BAI}$ e $\geq 12$ para BDI. Pontuações menores que essas representam níveis mínimos da sintomatologia avaliada em ambos os questionários, e os valores iguais ou superiores podem apresentar níveis leves, moderados ou graves.

O projeto foi avaliado e aprovado pelo Comitê de Ética em Pesquisa da Universidade Federal de Sergipe, CASE 42025015.7.0000.5546.

\section{RESULTADOS}

Responderam ao questionário 169 estudantes, entre eles 107 mulheres e 62 homens, com a mediana de idade de 21 anos. Foi percebido que 98,8\% (167) fazem uso diário de internet e/ ou redes sociais, dos quais 47,3\% (61) elegeram a utilização de aplicativos de mensagem instantânea como principal motivo que os faz acessar a internet, enquanto 22,5\% (29) afirmaram que o principal motivo são pesquisas, 18,6\% (24) redes sociais, $8,5 \%$ (11) entretenimento, e 3,1\% (4) notícias. Entre várias redes sociais e aplicativos de conversação instantânea, o WhatsApp foi o primeiro da lista quanto à frequência de uso em $94,4 \%$ dos casos.

$\mathrm{O}$ acesso à informação foi o principal benefício atrelado ao uso da internet em 48,7\% (76) dos casos, seguido da facilidade de comunicação em 44,2\% (69), lazer 6,4\% (10) e conhecer novas pessoas $0,6 \%$ (1). Além desses, vários benefícios secundários foram apontados como associados ao uso da internet, como, por exemplo, 79,3\% (134) relacionados ao estudo/trabalho, 61,5\% (104) a maior sociabilidade, $58 \%$ (98) a criar e manter contatos, $37,3 \%$ (63) a acesso à cultura, 6,5\% (11) a autoestima elevada, e $1,8 \%$ (3) não viram as opções anteriores como benefícios.

Por outro lado, os respondentes também apontaram interferências negativas do uso da internet nos seguintes quesitos: família, 21,2\% (34); lazer, 8,1\% (13); estudos, 67,5\% (108); atividade física, 31,7\% (51); alimentação, 5,6\% (9); relacionamentos amorosos, 6,9\% (11); relacionamentos entre amigos, 8,7\% (14); e apenas 9,9\% (16) negaram essas interferências.

O Gráfico 1 mostra a distribuição do tempo estimado gasto na internet. Não foi encontrada associação estatística entre esses dados e a presença de sintomas ansiosos e depressivos com os escores BAI ( $p=0,167)$ e BDI $(p=1,000)$.

No presente trabalho, foram investigados nos participantes alguns indícios da necessidade de verificação de internet. Além de alguns desses comportamentos apresentarem prejuízos na vida diária do indivíduo, foram encontradas algumas correlações com sintomas ansiosos e depressivos. Os resultados foram sumarizados na Tabela 1. 

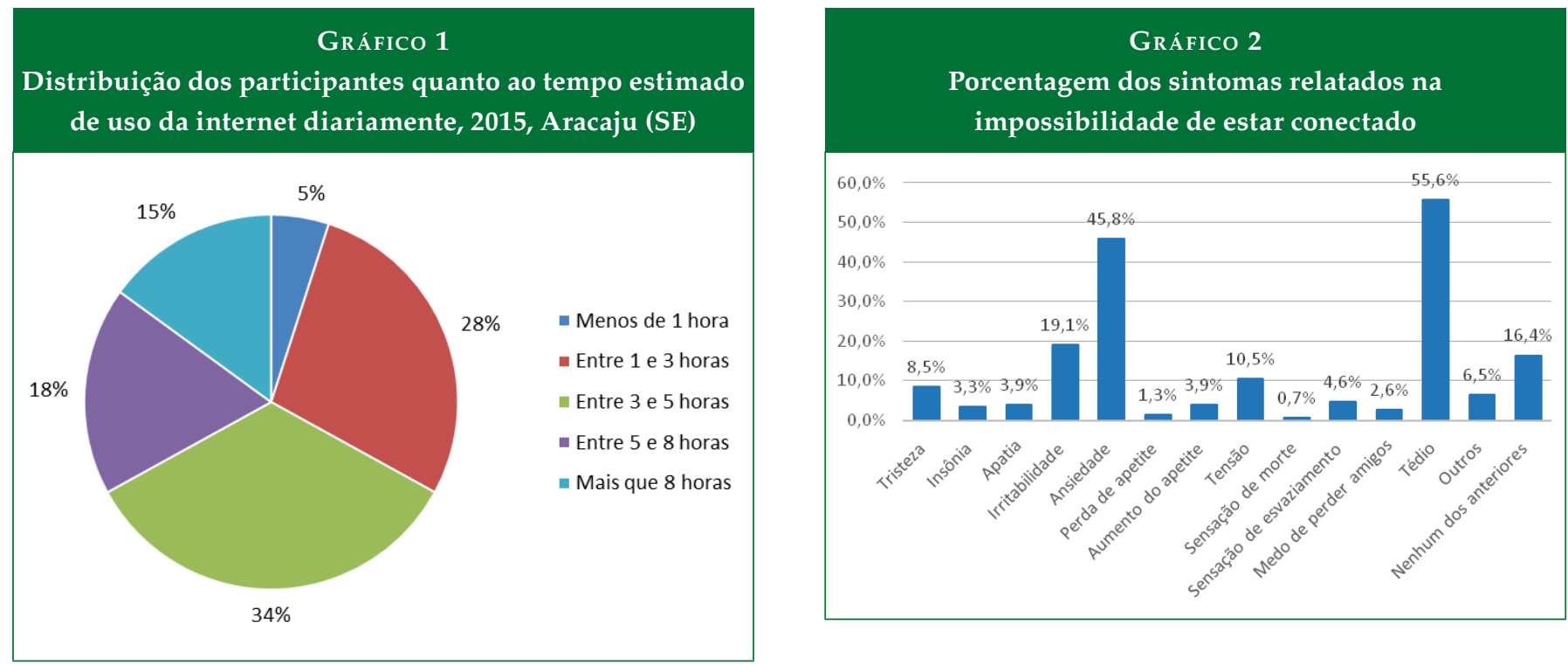

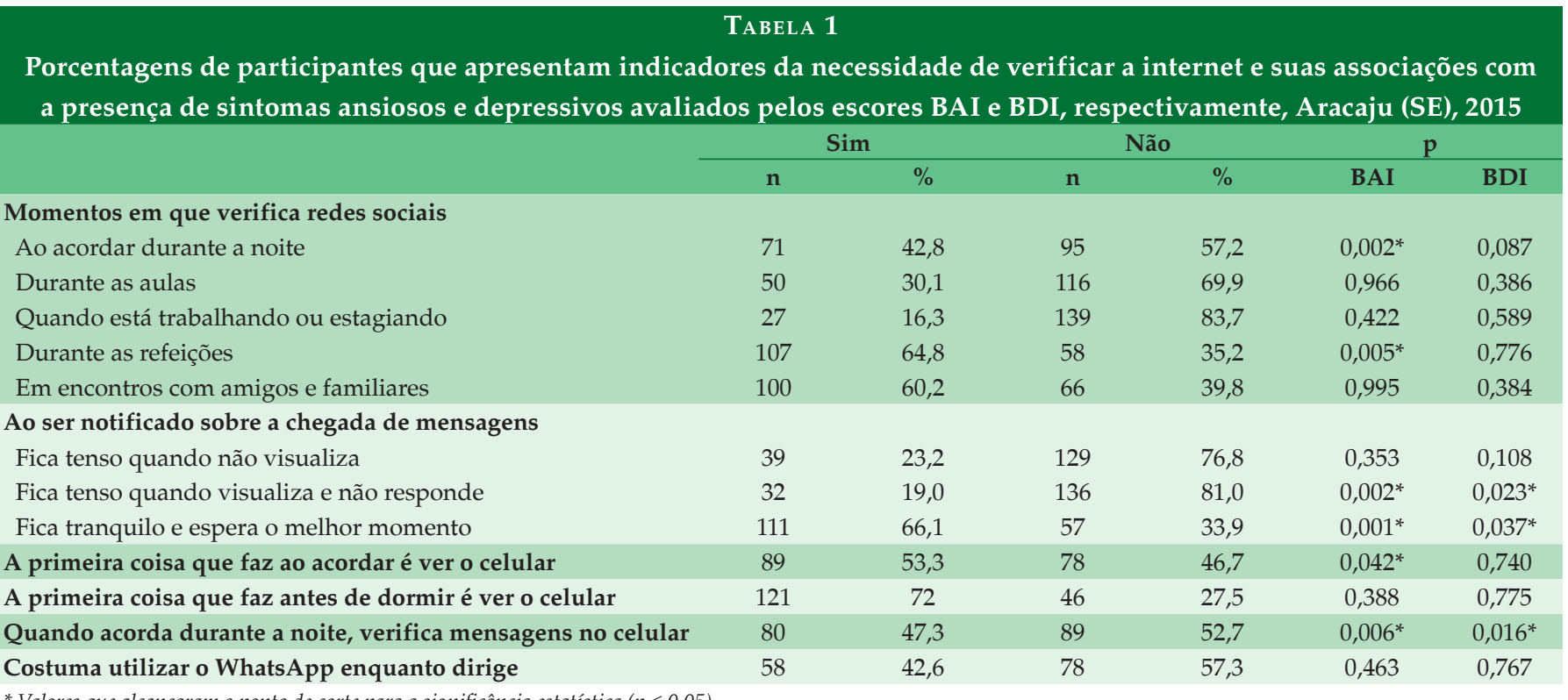

* Valores que alcançaram o ponto de corte para a significância estatística $(p \leq 0,05)$.

O Gráfico 2 mostra a frequência dos sintomas relatados na impossibilidade de estarem conectados à internet. Somente apresentaram associação com o escore BDI os que mostraram irritabilidade ( $p=0,015)$, tensão $(p=0,013)$ e sensação de esvaziamento ( $p=0,023)$. Em associação com o escore BAI, foram encontrados sintomas de tensão $(p=0,009)$ e sensação de esvaziamento ( $\mathrm{p}=0,043)$.

A frequência com que terceiros se queixam do tempo gasto na internet esteve estatisticamente relacionada com os valores encontrados no BAI ( $p \leq 0,001)$ e no BDI ( $p \leq 0,001)$. Ocorreu associação semelhante entre aqueles que tentam esconder o tempo que passam na internet e os escores BAI ( $p \leq 0,001)$ e $\operatorname{BDI}(p=0,006)$, mas esta associação não foi encontrada entre aqueles que tentam esconder de terceiros o que faziam na internet ( $\mathrm{p}=0,244$ para o BAI e $\mathrm{p}=0,194$ para o BDI).

Por fim, foram encontradas associações entre sintomas ansiosos e depressivos com efeitos prejudiciais e desadaptativos do uso da web. Entre aqueles que afirmaram que o uso do aplicativo de mensagens instantâneas WhatsApp prejudicava o rendimento acadêmico, foi encontrada relação significativa com o escore BAI ( $p=0,029)$, mas não foi encontrada com o BDI $(p=0,892)$. Entre aqueles que costumam perder o sono 
ou dormir menos por conta do uso excessivo da internet, foi encontrada associação com valores do BAI $(p=0,008)$ e do BDI $(\mathrm{p}=0,041)$.

\section{DISCUSSÃO}

Na atual pesquisa, 98,8\% (167) dos estudantes fazem uso diário de internet e/ou redes sociais, e quase a metade deles afirmou que utiliza como principal motivo de acesso os aplicativos de mensagem instantânea. A internet favorece a comunicação e a busca de informações e além disso é uma importante ferramenta de contato social ${ }^{12,13}$. Desde a década de 1990, a internet tornou-se parte essencial do cotidiano ${ }^{15}$. Assim, surge uma pressão do círculo social para estar constantemente conectado, tornando frequente o uso de redes sociais e aplicativos de mensagens instantâneas, como o WhatsApp ${ }^{14}$.

Desta forma, a dependência de internet apresenta índices que variam de $1 \%$ a $22,9 \%$ ao redor do mundo, sendo a maior parte das pesquisas voltadas para adolescentes e jovens adultos $^{5}$. A prevalência na Europa encontra-se entre 1-9\%, no Oriente Médio 1-12\% e na Ásia 2-18\% ${ }^{3}$.

Enquanto o tempo gasto online torna-se cada vez mais excessivo, o bem-estar psicológico de alguns usuários da internet está sendo prejudicado ${ }^{15}$. No presente estudo, não foi significativa a associação entre a quantidade de tempo gasto na internet e a presença de sintomas ansiosos e depressivos. Os jovens parecem acessar a internet principalmente por motivos positivos (ascensão social), enquanto os motivos negativos (autoafirmação) parecem ter menos importância ${ }^{16}$.

Dessa forma, a presença de sintomas tanto ansiosos quanto depressivos pode ser gerada pelo mau uso da internet e/ ou redes sociais, ou os sintomas podem estar presentes e o uso desta representa somente um mecanismo de compensação ${ }^{17}$. Segundo Dong et al. ${ }^{8}$ e Wallace ${ }^{18}$, não está claro se essa sintomatologia é precursora da problemática ou se é sequela. Provavelmente, a relação entre o uso da internet e a maneira como a ansiedade é gerenciada pode ser mais importante do que o nível da ansiedade em si ${ }^{19}$. Como Bischof-Kastner et al. ${ }^{16}$ afirmam, "o acesso à internet levado por motivos internos de regulação emocional é mais disfuncional do que o uso social da internet, o que parece mais recreativo" (p. 6).

Diferentes investigações chegam à conclusão de que a AI, muitas vezes, está relacionada a transtornos mentais, especialmente depressão e ansiedade $\mathrm{e}^{1,6,8,15}$. A depressão é a doença psiquiátrica mais relatada quando associada ao uso problemático da internet em adolescentes e ao aumento de depressão em adultos $2,5,20,21$. Um estudo recente apontou que o aumento da depressão está relacionado à maior utilização do tempo despendido na internet. No entanto, os autores não elucidam se a depressão precede essa dependência da internet ou se é uma consequência ${ }^{12}$. Diversas significâncias foram encontradas no presente estudo, como depressão e ansiedade associadas a indícios de uso inadequado da internet.

A problemática com o uso da internet pode ser associada ao comportamento de dependência, sendo mais suscetível em indivíduos que buscam estimulação externa e expressam mais intolerância ao tédio ${ }^{22}$. Segundo Elhai et $a l .{ }^{1}$, a dependência caracteriza-se pelo comportamento compulsivo no desejo de buscar sentimentos positivos, como um processo de aprimoramento do humor, podendo ser prejudicial ou adaptativo.

Alguns pesquisadores consideram a dependência da internet um transtorno de controle dos impulsos que afeta diretamente a qualidade de vida, causando consequências nas relações sociais, intolerância e sintomas de abstinência ${ }^{23}$. No estudo atual, os sintomas que apresentaram associação significativa com os escores de BAI e BDI relatados quando o estudante não tem a possibilidade de estar conectado foram sensação de vazio e tensão. Quanto à irritabilidade, 19,1\% relataram estar associada apenas ao BDI. O desejo de permanecer constantemente conectado parece impulsionar o uso excessivo das redes sociais ${ }^{1}$.

Dessa forma, estar conectado facilita o comportamento de verificar as notificações das redes sociais. Isso reforça uma sensação de segurança social com os amigos e parceiros, chamada de "busca de reafirmação" relacionada com o medo da perda $^{1}$. A incapacidade de controlar o uso da internet pode levar a sentimentos de angústia e comprometimento funcional das atividades cotidianas ${ }^{8}$. Reforçando tais resultados, os estudantes da presente pesquisa que ficaram tranqüilos, esperando o melhor momento para responder às notificações de mensagens, são aqueles com sintomas mínimos ou sem sintomas de ansiedade e depressão.

Os transtornos mentais mais comuns em estudantes de Medicina descritos na literatura são ansiedade e depressão e estão intimamente relacionados ${ }^{9,10,24}$. A combinação entre sobrecarga de estudos e treinamentos frequentes pode gerar um comportamento de compensação para criar meios de enfrentar esses sintomas ${ }^{25}$. A maneira como o jovem lida com a própria saúde e a de seus futuros pacientes tem influência dos aspectos cognitivos, comportamentais e das alterações emocionais quando não tratadas ${ }^{25}$. Assim, a presença de sintomas pode ser considerada um motivo para intervenção e cuidado do estudante. Podem-se considerar como fatores de risco que contribuem para a instabilidade emocional: sintomas ansiosos e pessoas inibidas, melancólicas e/ou com baixa autoesti$\mathrm{ma}^{15,18}$. Diferentes estudos associam tais fatores a altas taxas de queixas psicossomáticas e a sintomas psicopatológicos ${ }^{15}$. 
O uso excessivo da internet pode levar a diversos problemas, como mau gerenciamento do tempo, prejuízos físico-psicológicos e conflitos nas atividades diárias ou nos relacionamentos com amigos e familiares ${ }^{7,26,27}$. Ao usar o tempo durante o qual estuda ou dorme para ficar conectado, o estudante se torna suscetível a mudanças de humor e a vários transtornos mentais ${ }^{26}$.

No corrente trabalho, apenas 9,9\% dos participantes referiram ausência de interferências negativas do uso da internet em suas vidas. Num estudo na Alemanha, 9,3\% dos universitários relataram ter pelo menos uma consequência do uso da internet, destacando a negligência com o lazer e problemas em relacionamentos com família/parceiro(a), trabalho ou educação e saúde ${ }^{8}$. O presente estudo ratificou o achado acima nos itens lazer $(8,1 \%)$, família $(21,2 \%)$, relacionamentos amorosos $(6,9 \%)$ e entre amigos (8,7\%) e estudos $(67,5 \%)$.

A diminuição dos períodos de sono devido ao uso excessivo das redes sociais tem levado a uma piora no rendimento acadêmico $^{28}$. Na presente pesquisa, houve associação significativa entre a perda de sono pelo uso excessivo da internet e a presença de sintomas ansiosos e depressivos.

Em relação ao desempenho acadêmico, alguns estudos têm demonstrado que o aumento da quantidade de horas em que os estudantes ficam conectados na internet está relacionado com uma piora no aspecto em questão ${ }^{26}$. Quando o uso da internet acarreta dependência, ou seja, algo prejudicial, há uma queda nesse rendimento ${ }^{2,21,28,29,30}$. O atual trabalho revela a presença de sintomas ansiosos entre os estudantes que afirmaram que o uso do aplicativo de mensagem instantânea (WhatsApp) compromete o rendimento acadêmico.

\section{CONCLUSÃO}

A internet faz parte da vida hodierna, sendo utilizada das mais diversas maneiras a fim de preencher as necessidades de cada indivíduo. Entretanto, quando o usuário faz uso compulsivo da internet, despendendo muitas horas do seu dia conectado, tem-se a Adicção por Internet, que acarreta efeitos negativos em sua saúde mental.

Acadêmicos de Medicina, que naturalmente vivem sob constante pressão devido às responsabilidades inerentes ao curso, apresentam-se, conforme apontado por meio deste estudo, sob o espectro dos aspectos negativos que o uso excessivo da internet pode representar, denotado em sintomatologia ansiosa e depressiva, e prejudicando o rendimento acadêmico. É necessária uma avaliação criteriosa do corpo discente de modo a buscar tratamento eficaz para este transtorno.

\section{REFERÊNCIAS}

1. Elhai JD, Dvorak RD, Levine JC, Hall BJ. Problematic smartphone use: A conceptual overview and systematic review of relations with anxiety and depression psychopathology. Journal of Affective Disorders 2016; 207: 251-259.

2. Mazhari S. The Prevalence of Problematic internet Use and the Related Factors in Medical Students, Kerman, Iran. Addict Health, Summer \& Autumn 2012; 4 (3-4): 87-94.

3. Christakis AD. internet addiction: a 21st century epidemic? BMC Medicine [on line] 2010, 8:61 [capturado 10 de novembro 2016]; 1-3. Disponível em:http:/ /bmcmedicine. biomedcentral.com/articles/10.1186/1741-7015-8-61

4. Hinic D. Problems with 'internet addiction' diagnosis and classification. Psychiatria Danubina 2011; 23(2), 145-151.

5. Ko CH, Yen JY, Yen CF, Chen CS, Chen CC, et al. The association between internet addiction and psychiatric disorder: A review of the literature. European Psychiatry 2012; (27) 1-8.

6. Younes F, Halawi G, Jabbour H, El Osta N, Karam L, Hajj A, et al. internet Addiction and Relationships with Insomnia, Anxiety, Depression, Stress and Self-Esteem in University Students: A Cross-Sectional Designed Study. PLoS ONE [on line] 2016; [capturado 08 de novembro 2016]; 1-13.Disponível em:http://journals.plos.org/plosone/ article?id=10.1371/journal.pone.0161126

7. Akin A. The Relationships Between internet Addiction, Subjective Vitality, and Subjective Happiness. Cyberpsychology, Behavior, and Social Networking 2012; 15(8), 404-10.

8. Dong G, LU Q, ZHOU H, ZHAO X. Precursor or sequela: pathological disorders in people with internet addiction disorder. PLoS ONE [on line] 2011; [capturado 08 de novembro 2016]; 6(2) 1-5. Disponível em: http://journals.plos. org/plosone/article?id=10.1371/journal.pone.0014703

9. Oliveira, EN. Prevalência de sintomas depressivos em estudantes de Medicina da Universidade Federal da Bahia. Salvador; 2013. Monografia (Conclusão de curso) - Universidade Federal da Bahia.

10. Vasconcelos TC, Dias BRT, Andrade LR, Melo GF, Barbosa L, Souza E, el al. Prevalência de Sintomas de Ansiedade e Depressão em Estudantes de Medicina. Revista Brasileira de Educação Médica 2015; 39(1): 135-142.

11. Young KS. internet Addiction: The Emergence of a New Clinical Disorder. CyberPsychology \& Behavior 2009; 1(3): 237-44

12. Abreu CN, Karam RG, Góes DS, Spritzer DT. Dependência de internet e de jogos eletrônicos: uma revisão. Rev Bras Psiquiatr. 2008; 30(2): 156-67. 
13. Guedes E, Nardi AE, Guimarães FMCL, Machado S, King ALS. Social networking, a new online addiction: a review of Facebook and other addiction disorders. Medical Express [on line] 2016; [capturado 03 setembro 2016]; 3(1) 1-6.

14. Ho RC, Zhang MWB, Tsang TY, Toh AH, Pan F, Lu Y, et al. The association between internet addiction and psychiatric co-morbidity: a meta-analysis. BMC Psychiatry 2014; [capturado em 10 outubro de 2016]; 14(183): 1-10. Disponível em:http:/ /www.biomedcentral.com/1471-244X/14/183

15. Müller KW, Koch A. Dickenhorst U, Beutel ME, Duven E,1 Wölfling K. Addressing the Question of Disorder-Specific Risk Factors of internet Addiction: A Comparison of Personality Traits in Patients with Addictive Behaviors and Comorbid internet Addiction. BioMed Research International 2013, 2013: 1-7.

16. Bischof-Kastner C, Kuntsche E, Wolstein J. Identifying Problematic internet Users: Development and Validation of the internet Motive Questionnaire for Adolescents (IMQ-A). J Med internet Res, out 2014; [capturado 30 de outubro 2016]; 16(10). Disponível em:https://www.ncbi.nlm.nih. gov/pmc/articles/PMC4210952/?report=printable

17. Menezes PP. O virtual, o homem e a Psicanálise. Revista de Psicanálise Reverie 2012 do Grupo de Estudos de Fortaleza; 5(1): 100-09.

18. Wallace P. internet addiction disorder and youth. EMBO reports 2014; [capturado 30 de outubro 2016]; 15(1): 12-16. Disponível em:http://onlinelibrary.wiley.com/ doi/10.1002/embr.201338222/epdf

19. Marafanti I, D’Elia G, Pinheiro MCP, Cordeiro Q, Alves TCTF. Influência de sintomas ansiosos no desempenho acadêmico de formandos de medicina. Arq Med Hosp Fac Cienc Med Santa Casa São Paulo 2013: 1-6.

20. Çardak M. Psychological well-being and internet addiction among University students. TOJET: The Turkish Online Journal of Educational Technology - July 2013; [capturado 20 de outubro 2016]; 12(3): 134-141. Disponível em: http:/ / www.tojet.net/articles/v12i3/12312.pdf

21. Tsitsika A, Critselis E, Louizou A, Janikian M, Freskou A, Marangou E et al. Determinants of internet Addiction among Adolescents:A Case-Control Study. The Scientific World JOURNAL 2011; 11, 866-874.

22. Shi J, Chen Z, Tian M. internet Self-Efficacy, the Need for Cognition, and Sensation Seeking as Predictors of Problematic Use of the internet.Cyberpsychology, Behavior, and Social Networking, 2011: 14(4); 231-34.

23. Alavi SS, Maracy MR, Jannatifard F, Eslami M. The effect of psychiatric symptoms on the internet addiction disorder in Isfahan's University students.J Res Med Sci 2011
Jun; [capturado 15 de outubro 2016]; 16(6): 793-800. Disponível em:https://www.ncbi.nlm.nih.gov/pmc/articles / PMC3214398/?report=classic

24. Tabalipa FO, Souza MF, Pfützenreuter G, Lima VC, Traebert E, Traebert J. Prevalence of Anxiety and Depression among Medical Students. Revista Brasileira de Educação Médica 2015; 39(3): 388-394.

25. Baldassin S. Ansiedade e Depressão no Estudante de Medicina: Revisão de Estudos Brasileiros. Cadernos ABEM 2010; [capturado 15 de outubro 2016]; 6: 19-26. Disponível em:http:/ / docplayer.com.br/14923626-Ansiedade-e-depressao-no-estudante-de-medicina-revisao-de-estudos-brasileiros.html

26. Khoshakhlagh H, Faramarzi S. The Relationship of Emotional Intelligence and Mental Disorders with internet Addiction in internet Users University Students. Addict Health, Summer \& Autumn 2012; 4(3-4): 133-40.

27. Wang H, Zhou X, Lu C, Wu J, Deng X, Hong L. Problematic internet Use in High School Students in Guangdong Province, China. PLoS ONE 2011; 6(5); e19660: 1-8. doi: 10.1371/journal.pone.0019660

28. Andreassen CS. Development of a facebook addiction scale. Psychological Reports 2012; 110(2): 501-51.

29. Kuss DJ, Griffiths MD. Online Social Networking and Addiction - A Review of the Psychological Literature. Int. J. Environ. Res. Public Health 2011; 8: 3528-52. doi:10.3390/ ijerph8093528

30. Thomée S, Härenstam A, Hagberg M. Computer use and stress, sleep disturbances, and symptoms of depression among young adults - a prospective cohort study. BMC Psychiatry 2012; [capturado em 15 outubro de 2016]; 12(176): 1-14. Disponível em:http://bmcpsychiatry.biomedcentral.com/articles/10.1186/1471-244X-12-176

\section{CONTRIBUIÇÃO DOS AUTORES}

Maíra Sandes Moromizato: criação da ideia que originou o trabalho e elaboração de hipóteses; estruturação do método do trabalho; revisão de literatura; coleta de dados; escrita do manuscrito

Danilo Bastos Bispo Ferreira: estruturação do método do trabalho; revisão de literatura; coleta de dados; escrita do manuscrito

Lucas Santana Marques de Souza: estruturação do método do trabalho; revisão de literatura; coleta de dados; escrita do manuscrito

Renata Franco Leite: estruturação do método do trabalho; revisão de literatura; coleta de dados; escrita do manuscrito 
Fernanda Nunes Macedo: estruturação do método do trabalho; revisão de literatura; coleta de dados; escrita do manuscrito

Déborah Pimentel: criação da ideia que originou o trabalho e elaboração de hipóteses; orientação do trabalho; coordenação do grupo que realizou o trabalho; resolução de problemas fundamentais do trabalho; orientação da redação do manuscrito

\section{CONFLITO DE INTERESSES}

Não há conflitos de interesses.

\section{ENDEREÇO DE CORRESPONDÊNCIA}

\section{Maíra Sandes Moromizato}

Universidade Tiradentes - Medicina

Av. Murilo Dantas, 300

Farolândia - Aracaju

CEP 49032-490 - SE

E-mail: maia_moromizato@hotmail.com 